\title{
Molecular line observations and chemical modelling of galactic edge clouds
}

\author{
Paul Ruffle ${ }^{1}$, Tom Millar ${ }^{2}$, Helen Roberts ${ }^{2}$, \\ Don Lubowich ${ }^{3}$, and Christian Henkel ${ }^{4}$ \\ ${ }^{1}$ National Radio Astronomy Observatory, Green Bank, WV, USA \\ email: pruffle@nrao.edu \\ ${ }^{2}$ School of Mathematics and Physics, The Queen's University, Belfast, UK \\ ${ }^{3}$ Department of Physics and Astronomy, Hofstra University, Hempstead, NY, USA \\ ${ }^{4}$ Max-Planck-Institut für Radioastronomie, Bonn, Germany
}

\begin{abstract}
Edge Clouds 1 and 2 (EC1 and EC2) are large molecular clouds with the largest galactocentric distances known to exist in the Milky Way. We present observations of these clouds and use them to determine physical characteristics. For EC2 we calculate a gas temperature of $20 \mathrm{~K}$ and a density of $n\left(\mathrm{H}_{2}\right) \sim 10^{4} \mathrm{~cm}^{-3}$. Based on our $\mathrm{CO}$ maps, we estimate the mass of EC2 at around $10^{4} \mathrm{M}_{\odot}$, and continuum observations suggest a dust-to-gas mass ratio as low as 0.001. Chemical models have been developed to reproduce the abundances in EC2 and they indicate that: heavy element abundances may be reduced by a factor of five relative to the solar neighbourhood (similar to dwarf irregular galaxies and damped Lyman alpha systems); very low extinction $\left(A_{\mathrm{V}}<4 \mathrm{mag}\right)$ due to a very low dust-to-gas ratio; an enhanced cosmic ray ionisation rate; and a higher UV field compared to local interstellar values. The reduced abundances may be attributed to the low level of star formation in this region and are probably also related to the continuing infall of low metallicity halo gas since the Milky Way formed. We find that shocks from an old supernova remnant may have determined the morphology and dynamics of EC2, including the recently discovered star clusters embedded in the northern and southern cores. However, compared to EC2, EC1 appears to be a chemically less varied environment. The apparent molecule-poor nature of EC1 demonstrates the characteristics of clouds that have not had the benefit of SN shocks to stimulate an active cloud chemistry and star formation.
\end{abstract}

Keywords. Astrochemistry, dust, extinction, ISM: clouds, ISM: molecules, radio lines: ISM

Observations of $\mathrm{CO}$ emission at large galactocentric distances have detected a number of molecular clouds, including Edge Cloud 2 (EC2), with an estimated kinematic galactocentric distance of $R_{\mathrm{gc}} \sim 28 \mathrm{kpc}$ (Digel et al. 1994). EC2 appears to be up to $6 \mathrm{kpc}$ further away than EC1, the next most distant cloud, and much further than the extent of the optical disk of the Milky Way, $\sim 15-19 \mathrm{kpc}$ (Robin et al. 1992). EC2 has an effective radius of $\sim 20 \mathrm{pc}$ and is situated $\sim 360 \mathrm{pc}$ below the distant warped Galactic plane (Digel et al. 1996b). The CO luminosity of EC2 is at least a factor of 2 larger than those of the other 10 edge clouds, and is comparable to that of the Taurus Giant Molecular Cloud. EC2 is also the only edge cloud detected in the high-density tracer CS (Digel et al. 1996a), with CO maps showing that it has sub-structure.

EC2 was also found to have an associated H II region excited by an early B star (MR1) (de Geus et al. 1993). Spectra of MR1 (Smartt et al. 1996) indicate significant metal depletion, with elemental abundances reduced on average by some 0.5 dex. Subsequently, metal depletions of about five for $\mathrm{C}, \mathrm{N}$, and $\mathrm{O}$ have been calculated (Rolleston et al. 2000). NIR observations reveal young stellar objects in EC2 (Kobayashi \& Tokunaga 2000), and it is thought to be the most distant cloud in the Milky Way with evidence for star formation (Snell et al. 2002). More recently, two embedded young star clusters 
have been associated with EC2 and identified as T Tauri associations (Yasui et al. 2008). EC2 is also associated with the approaching side of the H I shell from supernova remnant (SNR) GSH 138-01-94 (Stil \& Irwin 2001), setting $R_{\mathrm{gc}} \sim 24 \mathrm{kpc}$ for EC2.

We have searched for line and continuum emission towards EC2 in order to constrain its physical conditions and chemical composition (Ruffle et al. 2007). A temperature of $20 \mathrm{~K}$ was estimated from our detections of ammonia and a gas density of $n\left(\mathrm{H}_{2}\right) \sim 10^{4} \mathrm{~cm}^{-3}$ was determined by comparing LVG models to our deconvolved line detections. Taking the clumpy structure of EC2 into account, we also calculated $M_{\mathrm{EC} 2} \sim 10^{4} \mathrm{M}_{\odot}$, and from the peak continuum emission we calculated a dust-to-gas mass ratio $\geqslant 0.001$.

To establish the most likely chemical and physical properties of EC2, we made a pseudo-time-dependent chemical kinetic model. This uses our observationally derived temperatures and densities and varies elemental initial abundances, photon flux (UV), cosmic ray ionisation $(\mathrm{CRI})$ rate and gas-to-dust ratio $\left(A_{\mathrm{V}}\right)$, in an attempt to fit the observed results. We found that heavy elements may be depleted by about a factor of five relative to local molecular clouds. The models also suggest a high UV photon field (10-20 $\times$ local values), with values of $A_{\mathrm{V}}$ up to $4 \mathrm{mag}$. Gas densities much above $n\left(\mathrm{H}_{2}\right)=1.2 \times 10^{4} \mathrm{~cm}^{-3}$ are excluded by the models, even if the UV field is increased. Some of our models indicate that steady-state is reached very quickly after $\sim 5,000$ years.

Sulfur-bearing molecules appear to be very over-abundant by at least an order of magnitude compared to local dark clouds. The observed high abundances of the radicals $\mathrm{C}_{2} \mathrm{H}$ and $\mathrm{CN}$ are typical of photon-dominated regions (PDRs). This may be related to a large value of the UV flux to grain surface area when compared to local clouds. In particular, we found that our best-fit models are consistent with reduced elemental abundances and a low dust-to-gas mass ratio. Such reduced abundances may be attributed to the low level of star formation in this region, and are probably also related to the continuing infall of low metallicity halo gas since the Milky Way formed. In addition, although EC2 does contain young stars, there is no evidence of the late-type stars which produce dust grains, thereby justifying the assumption of a high ratio of UV flux to grain surface area. We conclude therefore, that despite the position of EC2 in the Galaxy, UV photons (rather than cosmic rays) play an important role in establishing its chemical composition.

Given that EC2 is in a region of extremely low gas pressure and very small spiral arm perturbation, the question remains as to the origin of the structure and chemistry in EC2. The SNR associated with EC2, GSH 138-01-94, consists of a H I shell with an expansion age of 4.3 Myr. We conclude that the formation, structure and chemistry of EC2, and subsequent star formation, may be the direct result of shock fronts from the SNR propagating through the medium sometime between 1,000 and 10,000 years ago.

\section{References}

de Geus, E. J., Vogel, S. N., Digel, S. W., \& Gruendl, R. A. 1993, ApJ (Letters), 413, L97

Digel, S., de Geus, E., \& Thaddeus, P. 1994, ApJ, 422, 92

Digel, S. W., de Geus, E. J., Henkel, C., et al. 1996a, in IAU Symp. 170, 20P

Digel, S. W., Hunter, S. D., Mukherjee, R., et al. 1996b, in IAU Symp. 170, 22

Kobayashi, N. \& Tokunaga, A. T. 2000, ApJ, 532, 423

Robin, A. C., Creze, M., \& Mohan, V. 1992, ApJ (Letters), 400, L25

Rolleston, W. R. J., Smartt, S. J., Dufton, P. L., \& Ryans, R. S. I. 2000, A\&A, 363, 537

Ruffle, P. M. E., Millar, T. J., Roberts, H., et al. 2007, ApJ, 671, 1766

Smartt, S. J., Dufton, P. L., \& Rolleston, W. R. J. 1996, A\&3A, 305, 164

Snell, R. L., Carpenter, J. M., \& Heyer, M. H. 2002, ApJ, 578, 229

Stil, J. M. \& Irwin, J. A. 2001, ApJ, 563, 816

Yasui, C., Kobayashi, N., Tokunaga, A. T., Terada, H., \& Saito, M. 2008, ApJ, 675, 443 